\title{
Application of high resolution acoustics for determination of the physical properties of fluid sediments
}

Coen WERNER, Netherlands

Senior Geologist STEMA Systems

Topic: H: Geophysics of the marine environment

\section{INTRODUCTION}

For study of areas with fluid sediments, often a traditional low frequency echosounder is used. However, often this appears not to be reliable. The results consist of a single depth digitalisation value depending on the echosounder settings. The information is difficult to validate, because digital raw data are not included, digitalisation often is not stable and no information about physical properties of the seabed is derived.

The integration of high resolution digital seismic acquisition techniques with low frequency echosounder frequencies provides extensive opportunities for validation of acquired (raw) data. After calibration with geophysical point measurements this type of high resolution acoustics also can be a useful tool for characterization of the physical properties of fluid sediments.

A geophysical point measurement device usually derives a vertical profile with physical parameters versus depth. An example is the RheoTune, which appplies the tuning fork method and produces density and yield strength. Another device is the nuclear transmission/ backscatter [Van Craenenbroeck et al, 1998], that measures density.

This paper presents a case study, that illustrates the application of high resolution digital seismics (Silas system) together with geophysical point measurements using a RheoTune.

The general procedure of used method is described, together with the results that give insight in the potential capability of high resolution acoustics.

It is clear that a more thorough understanding of the physical properties of fluid sediments is important for economical reasons, e.g. for maintenance of the navigable depth. Also this type of information gives extra input to the study of geomorphological dynamics in an area.

\section{AREA AND METHODS}

Area

The case study consists of 2 surveys in a channel of a tidal flat area in the Waddenzee (Northern Netherlands). The area can be characterized by significant tidal movements (between app. $+2 \mathrm{~m}$ $2 \mathrm{~m}$ ) and related strong tidal currents.

\section{Methods}

Each survey was carried out using a $24 \mathrm{kHz}$ low frequency transceiver combined with a high resolution digital seismic system (Silas EBP-10). Followed procedure is described by Fontein, Byrd [2008].

The complete raw signal was recorded.Subsequently geophysical point measurements were carried out using a RheoTune device (fig.1.) This device measures the in-situ density and yield stress of water and fluid sediment together with depth (pressure), temperature and sensor orientation. The in-situ density is measured using the tuning fork principle [Werner,Fontein, 2010]. In this type of measurement the resonance frequency of the Tuning Fork depends on the density of the medium. The amplitude of vibration is controlled by the yield stress of the medium. 
The surveys consist of a calibration survey and a regular survey. During the calibration survey in advance all parameters of the high resolution seismic system were determined during standard barcheck and soundvelocity probe procedures. Initially a number of high resolution seismic lines were run along the longitudinal axis of the area. Based on these recordings, subsequently locations were selected for geophysical point measurements which were carried out relatively shortly afterwards. The geophysical point measurements were situated preferably at locations with highest thickness development of fluid mud. At each of the selected points a depth/density/yield stress profile was obtained (fig.2).

The point measurements enabled the density calibration of the high resolution seismics. During this calibration at each location the seismic results (fig. 3A, 3B) are converted into a synthetical density profile (Fig. 3C) in an automated procedure by the software. This is done for different estimates of the arrival power of the signal, the power of the signal that arrives at the seafloor. The calibration ends at the arrival power where the match between the synthetical density profiles from seismic and geophysical point measurements (fig.3D) is optimal.

The synthetical densityprofiles are calculated using standard acoustic laws, which relate arrival signal power and reflected power to the physical properties of the sediment [McGee, 1992], which can be described by the impedance, see formula (1).

in which:

$$
\text { Impedance }=\rho * v
$$

$\rho=$ density of sediment layer in $\mathrm{kg} . / \mathrm{l}$.

$\mathrm{v}=$ propagation velocity of $\mathrm{p}$-waves in sediment in meters/second

After this calibration, the seismic system produces calibrated synthetic densityprofiles for each seismic shot (fig.3 C) and the depth of a specific densitylevel on any location on the seismic lines.

The calibration was re-used 3 weeks later during a regular survey with the same seismic system for determination of the physical properties of the seabed. During this survey also the same system settings were used. Extra geophysical point measurements provided a check for the accuracy of the calibration.

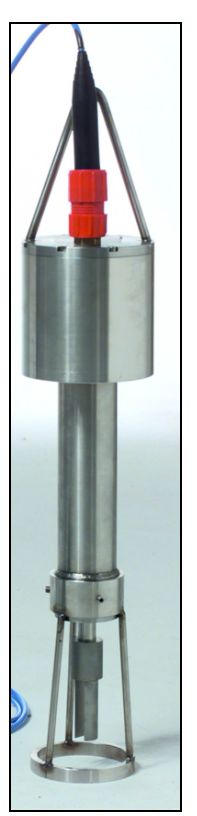

Fig. 1 RheoTune fluid

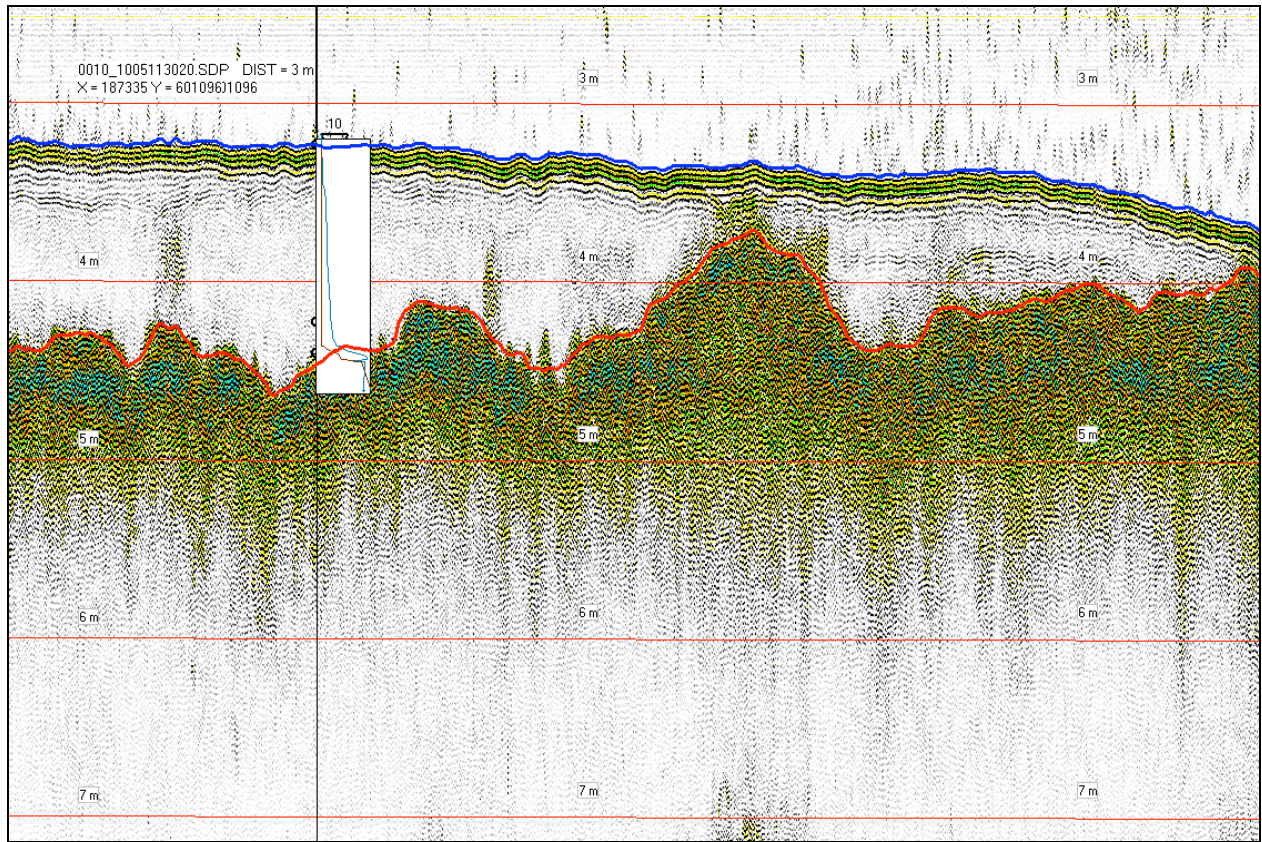

Fig. 2 : High resolution acoustical recording and location of a point measurement in sediment in area of case study. See Appendix for Legend. 


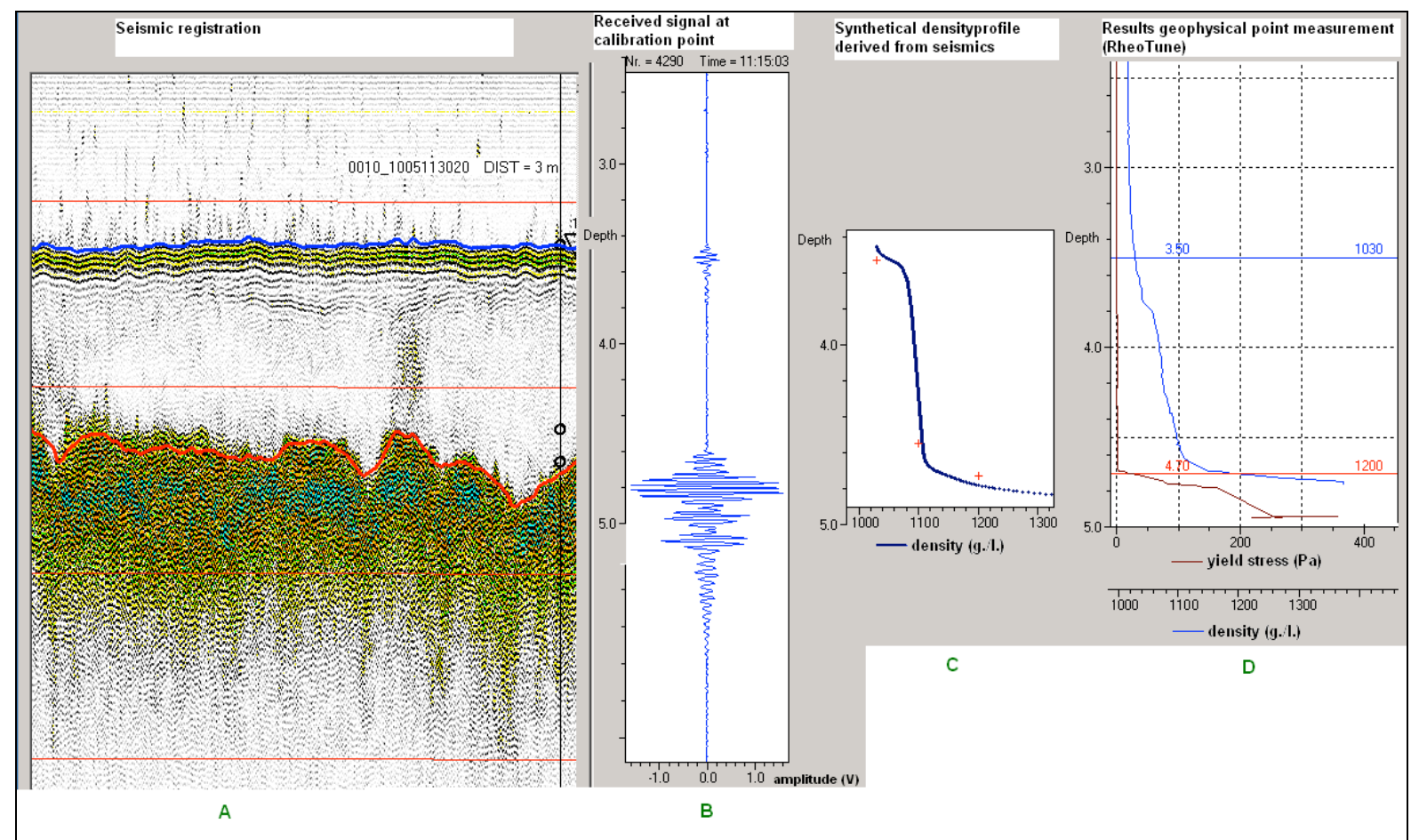

Fig.3 Procedure of density calibration of high resolution seismics as applied by Silas. $A=$ Seismic registration, $B=$ Received signal at calibration point, $C=$ Synthetical densityprofile derived from seismics at calibration point, $D=$ Results geophysical point measurement (RheoTune) 


\section{RESULTS}

Calibration survey

At the geophysical point measurement locations, high resolution seismic profiles and geophysical point measurements carried out in the same time interval (app. 90 minutes) provide two independend data sets for location of the top of the fluid sediment, the lutocline (table 1).

\begin{tabular}{|c|c|c|c|c|c|c|c|c|}
\hline $\begin{array}{l}\text { A } \\
\text { Location } \\
\text { number }\end{array}$ & $\begin{array}{l}\text { B } \\
\text { Time } \\
\text { difference } \\
\text { (minutes) }\end{array}$ & $\begin{array}{l}\text { C } \\
\text { Distance } \\
(\mathrm{m})\end{array}$ & $\begin{array}{l}\text { D } \\
\text { Depth } \\
\text { seismic } \\
\text { (m.to } \\
\text { N.A.P.) }\end{array}$ & $\begin{array}{l}\text { E } \\
\text { Depth } \\
\text { (Tune) } \\
\text { (m to } \\
\text { N.A.P.) }\end{array}$ & $\begin{array}{l}\text { F } \\
\text { Depth Difference } \\
\text { D-E (m) }\end{array}$ & $\begin{array}{l}\text { G } \\
\text { Arrival } \\
\text { Density } \\
\text { (g./l.). }\end{array}$ & $\begin{array}{l}\mathrm{H} \\
\text { Density } \\
\text { Gradient } \\
(\mathrm{g} . / \mathrm{l} / \text { per } \mathrm{cm})\end{array}$ & $\begin{array}{l}\text { I } \\
\text { Inter- } \\
\text { val } \\
\text { (m) }\end{array}$ \\
\hline 0001_759 & 63 & 2 & 3.58 & 3.47 & 0.11 & 1024 & 1.72 & 0.11 \\
\hline 0001_409 & 67 & 4 & 4.00 & 3.88 & 0.12 & 1020 & 5.38 & 0.13 \\
\hline $0003 \_657$ & 71 & 2 & 4.09 & 3.98 & 0.11 & 1021 & 8.7 & 0.07 \\
\hline 0006_924 & 41 & 2 & 3.40 & 3.51 & -0.11 & 1026 & 0.45 & 0.11 \\
\hline $0007 \_724$ & 37 & 3 & 3.42 & 3.45 & -0.03 & 1026 & 1.18 & 0.16 \\
\hline $0008 \_211$ & 40 & 0 & 3.29 & 3.22 & 0.07 & 1027 & 0.27 & 0.40 \\
\hline 0009_614 & 33 & 4 & 3.40 & 3.09 & 0.31 & 1023 & 0.55 & 0.25 \\
\hline $0010 \_020$ & 15 & 3 & 3.32 & 3.33 & -0.01 & 1024 & 0.42 & 0.28 \\
\hline \multirow[t]{2}{*}{ 0011_449 } & 34 & 1 & 3.53 & 3.51 & 0.02 & 1027 & 0.43 & 0.16 \\
\hline & & & & & StDev $=0.12$ & $\begin{array}{l}\text { Average }= \\
1024\end{array}$ & & \\
\hline
\end{tabular}

Table 1. Results of calibration survey: Top of fluid sediment

Explanation:

Column B: Time difference between seismic and geophysical point measurement (Minutes)

Column C: Distance between seismic and geophysical point measurement (m.)

Column D: Depth first reflector seismic (m. to N.A.P. )

Column E: Depth first density change according to geophysical point measurement (Tune) (m. to N.A.P.)

Column F: Difference between column D and $E(m$.)

Column G: Density above first density change in geophysical point measurement (g./l.).

Column H: Density gradient at first densitychange in geophysical point measurement in $\mathrm{g} . / \mathrm{l}$. per $\mathrm{cm}$.

Column I: Length of first densitychange $(\mathrm{m})$

The relatively small values in Column $\mathrm{F}$ of Table 1 show that the location of the first reflector in the seismics corresponds with the first densitychange in the geophysical point measurement. The small values also show that the densitychange was detected by the seismics. Because above data confirm close correspondence between first reflector and first densitychange it is valid to use these measurements for density calibration of the seismics (method described in Section "Area and Methods").

After calibration the geophysical point measurements provided an extra check for the accuracy of the $1200 \mathrm{~g} . /$. density level in seismic synthetical densityprofiles. The difference between densitylevels predicted by seismic and the level measured by the geophysical point measurement does not exceed $+-0.2 \mathrm{~m}$, with a standardeviation of $0.12 \mathrm{~m}$.. These variations are similar to observed variability at the lutocline (Table 1).

Regular survey

The regular survey was carried out after 3 weeks in the same area. The same system settings and the calibration described above were applied to measure the location of the $1200 \mathrm{~g} . / \mathrm{l}$. level.

Geophysical point measurements finally provided a check for accuracy and validity of the re-used density calibration (see table 2). The difference between $1200 \mathrm{~g} . / \mathrm{l}$. densitylevels predicted by seismic and the level measured by the geophysical point (Tune) measurement does not exceed $+-0.22 \mathrm{~m}$. (Standarddeviation $0.16 \mathrm{~m}$.). These values are slightly higher than during the calibration survey. Several point measurements could only be derived with time differences larger than 70 minutes and are consequently less representative. 


\begin{tabular}{|c|c|c|c|c|c|c|}
\hline $\begin{array}{l}\text { A } \\
\text { Location } \\
\text { number }\end{array}$ & $\begin{array}{l}\text { B } \\
\text { Time } \\
\text { difference } \\
\text { (minutes) }\end{array}$ & $\begin{array}{l}\text { C } \\
\text { Distance } \\
\text { Seismics } \\
\text { and point } \\
\text { measure- } \\
\text { ment } \\
\end{array}$ & $\begin{array}{l}\text { D } \\
\text { Depth } \\
1200 \mathrm{~g} . / \mathrm{l} . \\
\text { level seismic } \\
\text { (m. to } \\
\text { N.A.P. ) } \\
\end{array}$ & $\begin{array}{l}\text { E } \\
\text { Depth } \\
1200 \text { g./l. } \\
\text { level (Tune) } \\
\text { (m to } \\
\text { N.A.P.) } \\
\end{array}$ & $\begin{array}{l}\text { F } \\
\text { Depth Difference } \\
\text { synthetic } 1200 \\
\text { g./l. }-1200 \mathrm{~g} . / \mathrm{l} . \\
\text { (Tune) in m. }\end{array}$ & $\begin{array}{l}\text { G } \\
\text { Thickness } \\
\text { Fluid sediment above } \\
1200 \mathrm{~g} . / \mathrm{l} . \text { level in m. }\end{array}$ \\
\hline $005 \_607$ & 39 & 7 & 4.0 & 3.82 & 0.18 & 0.98 \\
\hline $003 \_848$ & -72 & 2 & 4.35 & 4.57 & -0.22 & 0.96 \\
\hline $001 \_453$ & -77 & 10 & 4.75 & 4.91 & -0.16 & 1.47 \\
\hline $06 \_214$ & -80 & 6 & 4.31 & 4.16 & 0.15 & 1.03 \\
\hline $011 \_632$ & 23 & 6 & 4.79 & 4.74 & 0.05 & 1.51 \\
\hline $012 \_438$ & 20 & 2 & 4.65 & 4.86 & -0.21 & 1.25 \\
\hline $013 \_242$ & 17 & 5 & 4.54 & 4.63 & -0.09 & 1.25 \\
\hline \multirow[t]{2}{*}{ 014_924 } & 13 & 2 & 4.70 & 4.70 & 0.00 & 1.38 \\
\hline & & & & & StDev $=0.16$ & \\
\hline
\end{tabular}

Table 2. Verification of results of regular survey: seismic synthetic $1200 \mathrm{~g}$./l. Levels compared with $1200 \mathrm{~g} . / \mathrm{l}$. levels resulting from geophysical point measurements (Tune). Smallest diferences are generally found at time differences under 24 minutes. Explanation:

Column D: Depth calibrated synthetic 1200 g./l. level seismic (m. to N.A.P. )

Results: Yield strength

The geophysical point measurements of the Tune indicate the yield strength of the fluid sediment is generally low and does not exceed 20 Pascal. The yield strength of the majority of the fluid sediment is lower than 5 Pascal. This makes the sediment more susceptible to currents and dynamic behaviour. In investigated area under the $1200 \mathrm{~g}$./l. density level often a sharp transition is observed to yield strengths exceeding $100 \mathrm{~Pa}$.

It is important to notice that a lower yield strength will have less effect on vessel manouvrability. Consequently the yield stress is also an important parameter for maintenance of the navigable depth. How to integrate Rheological parameters (such as yield stress and viscosity) as extra criteria beside density for navigability is still under investigation.

\section{DISCUSSION}

Calibration of seismic

At the top of fluidized sediments the lowest density gradient is $0.27 \mathrm{~g} . / \mathrm{l}$. (Table 1 ) which is detected by high resolution seismics. This is however a single observation and possibly due to time mismatch between seismic and geophysical point measurement. After rejection of this single value, table 1 confirms a seismic detection capability for densitygradients of at least $0.4 \mathrm{~g} . / \mathrm{l}$ per $\mathrm{cm}$.

There might be some influence on presented standdarddeviation values for accuracy of calculated synthetic densitylevels, because of:

- Time difference between seismic and geophysical point measurement

- Horizontal distance between location of geophysical point measurement and seismic line True values for accuracy could be better, but are difficult to verify. In order to improve seismic density calibrations presented a larger number of geophysical point measurements can be adopted. 
Area dynamics

Largest accummulations of fluid sediment occur in the $1.5 \mathrm{~km}$ long nearshore channel area in the center section. The situation however proved to be dynamic, because the surveyresults show significant changes in location of the top of fluid sediment layer after app. 90 minutes.

Short term changes at deeper levels in the fluid sediment such as the $1200 \mathrm{~g} . / \mathrm{l}$. density level, are less pronounced.

Most changes in the state of the fluid sediment layer could be related to sediment transport due to tidal currents.Occasionally also dramatic state changes in the fluid sediment were observed. In the majority of these occasions a relation with ship traffic was confirmed.

\section{CONCLUSIONS}

The presented information leads to following conclusions:

- The digital high resolution seismic registration provides more information than the results of a traditional echosounder and provides higher relability and more verification options.

- High resolution seismic proves to be a useful tool for characterization the physical properties of fluid sediments. The calibration survey indicates this type of the seismic can detect relatively small densitygradients of $0.4 \mathrm{~g}$./l. per $\mathrm{cm}$ in fluid sediment of low yield strength $(<5$ $\mathrm{Pa}$ )

- The application of the density calibration for the seismics proved to be valid for at least three weeks, with a standarddeviation of $0.16 \mathrm{~m}$. for the difference between $1200 \mathrm{~g} . / . \mathrm{l}$. seismic synthetical densitylevels and $1200 \mathrm{~g} . / . \mathrm{l}$. densitylevels of geophysical point measurements.

- The geophysical point measurements of the Tune indicate the yield strength of the fluid sediment in investigated area is generally below $20 \mathrm{~Pa}$. The yield strength of the majority of the fluid sediment is lower than 5 Pascal.

\section{REFERENCES}

1. K. van Craenenbroeck, J. Duthoo, M. Vandecasteele, J.A. Eygenraam and R. Van Oostveen, Application of Modern Survey Techniques in Today's dredging Practice (Terra et Aqua nr. 72, 1998). 6-8

2. W.F. Fontein and R.W. Byrd, The nautical depth approach, a review for implementation (WODCON XVIII Annual Dredging Seminar,2007)

3. R.G. Mc Gee, and Robert F. Ballard, An Acoustic impedance method for subbottom material characterization ( Hydraulic Engineering sessions at water forum, 1992).

4. C. J. Werner and W.F. Fontein,Tune systems: general principle of operation (STEMA, 2010) 
CONTACT DETAILS (of corresponding author only)

Coen WERNER

STEMA Systems b.v.

Poppenbouwing 52,

4190 CB Geldermalsen

NETHERLANDS

Tel.: +31345580395

Fax: +31345570649

Email:coen.werner@stema-systems.nl

Web site: www.stema-systems.nl 